\title{
La educación rural en tiempos de COVID-19. Experiencias de continuidad pedagógica en las escuelas primarias de Maipú, provincia de Buenos Aires, Argentina*
}

\author{
Gustavo Javier Annessi (1) ; Juan Ignacio Acosta (D) \\ ${ }^{1}$ Centro de Capacitación, Información e Investigación Educativa de Maipú (CIIE); ${ }^{2}$ Universidad Nacional de La \\ Plata (UNLP), Argentina
}

Resumen. Este trabajo presenta los resultados de una indagación realizada a los docentes de escuelas primarias localizadas en el espacio rural de Maipú, provincia de Buenos Aires, Argentina, acerca de las propuestas de enseñanza y medios de comunicación que han implementado para atender a la continuidad pedagógica en este actual contexto de aislamiento social, preventivo y obligatorio (ASPO), implementado desde mediados de marzo de 2020. Para garantizar las trayectorias educativas de los alumnos, el gobierno de la provincia de Buenos Aires puso en marcha un programa de continuidad pedagógica, con el objetivo de que los estudiantes desde sus hogares y con el acompañamiento de sus docentes, continúen con el proceso educativo. Los docentes de las escuelas rurales tienen una larga trayectoria en el desarrollo de una dinámica particular que les ha permitido hacer frente a este escenario con mayor celeridad, gracias a la experiencia adquirida en el trabajo con los alumnos y sus familias, donde éstas ya forman parte de la escuela, con tiempos prolongados de no presencialidad. Sin embargo, en estos espacios hay situaciones muy heterogéneas porque no todas las familias cuentan con el tiempo ni las competencias necesarias para poder acompañar la continuidad pedagógica. Sostener la educación a distancia se vuelve difícil debido a la falta de dispositivos móviles y/o escasa o nula señal de Internet

Palabras clave: escuela rural; educación primaria; continuidad pedagógica.

A educação rural na época do COVID-19. Experiências de continuidade pedagógica nas escolas primárias de Maipú, província de Buenos Aires, Argentina

Resumo. Este trabalho apresenta os resultados de uma pesquisa feita aos professores de escolas primárias, localizadas na área rural de Maipú, sobre as propostas pedagógicas e os meios de comunicação implementados para atender à continuidade pedagógica neste contexto atual de isolamento social, preventivo e obrigatório (ASPO), vivido desde meados de março de 2020. Os professores das escolas rurais têm uma longa trajetória de desenvolvimento de uma dinâmica particular, que Ihes permitiu enfrentar mais rapidamente este cenário, graças à experiência adquirida no trabalho com os alunos e suas familias, onde estas já fazem parte da escola, com longos períodos sem aulas presenciais. Entretanto, nestes espaços há situações muito heterogêneas, porque nem todos os pais, mães e famílias têm o tempo ou as competências necessárias para poder acompanhar a continuidade pedagógica e sustentar a educação à distância por períodos muito longos nestas situações. Também é muito difícil garantir a continuidade pedagógica com falta de dispositivos móveis e/ou com pouco ou nenhum sinal de internet.

Palavras-chave: escuela rural; educación primaria; continuidad pedagógica.

Rural education in times of COVID-19. Experiences of pedagogical continuity in the primary schools of Maipú, province of Buenos Aires, Argentina

Abstract. This paper presents the results of an inquiry made to teachers of primary schools located in the rural area of Maipú, province of Buenos Aires, Argentina, about the teaching and communication proposals that they have implemented to attend to the pedagogical continuity in this current context of social, preventive and compulsory isolation (ASPO), implemented since mid-March 2020. In order to guarantee the educational trajectories of the students, the government of the Province of Buenos launched a program of pedagogical continuity, with the aim of that students from their homes and with the accompaniment of their teachers, continue with the educational process. Teachers in rural schools have a long history in developing a particular dynamic that has allowed them to face this scenario more quickly, thanks to the experience acquired in working with students and their families, where they are already part from school, with long periods of absence. However, in these spaces there are very heterogeneous situations because not all families have the time or the necessary skills to be able to accompany the pedagogical continuity. Sustaining distance education becomes difficult due to the lack of mobile devices and / or little or no Internet signal.

Keywords: rural school; primary education; pedagogical continuity.

* Una versión preliminar y exploratoria de este artículo puede encontrarse en una compilación que está disponible en https://bit.ly/2RMNCbJ 


\section{Introducción}

\subsection{Pandemias y educación. Antecedentes de la situación actual.}

En el despliegue del sistema escolar de fines del siglo XIX y principios del siglo XX, las sociedades se propusieron la construcción de vínculos estrechos entre educación y salud basados en el saber científico. Se trata de vínculos que fueron cambiando con el tiempo y establecieron a la escuela como uno de los espacios privilegiados para potenciar dicho encuentro.

Desde el siglo XX encontramos a nivel nacional algunos antecedentes que tuvieron implicancias en el sistema educativo: la gripe española de 1918, el brote de poliomielitis de 1956 y la pandemia de gripe A (H1N1) de 2009.

En los años 1918 y 1919 Argentina fue azotada por la pandemia de gripe -o 'grippe', o 'influenza española', o 'dama española'-. Entre las medidas adoptadas fue el cierre de las escuelas durante 10 días, período en el que se "ha puesto en práctica lo mejor que la ciencia aconseja para tratar de disminuir su propagación como: ser cierre de escuelas, de espectáculos públicos en locales cerrados y hasta de impedir reuniones en los cafés hasta altas horas de la noche" (Bortz, 2017, p. 247).

Otro antecedente se da en el año 1956, donde perdieron la vida alrededor de 6.500 personas a causa de la poliomielitis. Ese año las clases no se iniciaron hasta finales de mayo, o incluso más tarde, de acuerdo con la provincia que se analice.

El antecedente más reciente fue en 2009 con una nueva gripe, el Influenza A (H1N1). La "Gripe A" hizo que los gobiernos de la provincia de Buenos Aires y de Capital Federal declarasen la emergencia sanitaria y la suspensión de clases, anticipando en dos semanas el receso escolar.

Así llegamos a la actual pandemia provocada por la COVID-19 en 2020, que provocó el cierre de las instituciones educativas de todos los niveles de la educación básica desde el lunes 16 de marzo y no se sabe -al momento de producir este documento- hasta qué fecha permanecerá esta situación. En todo este tiempo se configuró un sistema de enseñanza basado en la virtualidad.

\subsection{La educación rural en un contexto geográfico de despoblamiento}

Las escuelas en el área rural fueron creadas en un contexto de emergencia por la alfabetización en estas áreas, llamadas desfavorables en diferentes grados de acuerdo a su accesibilidad. A pesar del fuerte proceso de despoblamiento, la oferta de educación rural no disminuyó. Incluso aumentó en las últimas décadas con la creación de escuelas secundarias y jardines de infantes rurales. Si bien la mayoría de los establecimientos educativos presentan oscilaciones en su matrícula interanuales, e incluso estacionales, las familias que han formado parte de su comunidad educativa resisten a su manera a la absorción urbana.

En estos últimos 20 años se llevaron adelante ciertas políticas de sostenimiento de la educación rural que han apuntado a afrontar las diversas problemáticas de las zonas rurales. Una de ellas es la estrategia de "agrupamiento como herramienta estratégica para el desarrollo territorial y de Islas" (DGCyE, 2009, p. 9). La misma promueve el trabajo de instituciones y personas organizadas en red, de compartir lecturas de la 
realidad para problematizarla, y diseñar posibilidades para incidir en ella, gestionar y disponer de recursos de manera colectiva y complementaria. Este modo de trabajo, articulado y comprometido entre los distintos sectores sociales de las comunidades posibilita la superación del aislamiento con especial incidencia positiva en el medio rural y de islas.

El rol de los docentes rurales a lo largo de la historia ha sido fundamental para poder retener parte de la matrícula que asiste a estos establecimientos, muchas veces alejados de los centros urbanos más próximos, y cuyos procesos migratorios desde hace tres décadas se vienen dando de manera ininterrumpida:

La tensión que es necesario tener presente es que suelen ser las escuelas que se encuentran sometidas a las transformaciones demográficas y socioproductivas que generan la reducción de la población por abandono del campo. El análisis de la ratio docente/alumno puede determinar el riesgo de cierre con la consecuente desaparición de la garantía de escolarización (MEN, 2012, p. 16).

Los docentes rurales están acostumbrados a desarrollar estrategias de continuidad pedagógica en distintos momentos del año escolar. Por ejemplo, en aquellos años donde hay más lluvias que lo habitual se reducen significativamente los días efectivos de clases.

Las propuestas de continuidad garantizan que los alumnos que asisten a estos establecimientos tengan las mismas posibilidades de aprender que los alumnos de los centros urbanos y que los días que no asisten a la escuela continúen con sus aprendizajes. En la Argentina hay muchas y muy variadas situaciones que garantizan la continuidad pedagógica en contextos muy complejos, y que habilitan alternar períodos de asistencia a la escuela con estadías en la casa.

Una alternativa que se desarrolla en algunas zonas de población dispersa y localización de la escuela en territorios de difícil acceso o carencia de formas de traslado cotidianas son formas organizativas en las que alumnos y docentes conviven en la escuela con la jornada escolar extendida durante un cierto período y vuelven a sus hogares durante el período siguiente.

Se reconocen con expresiones del tipo 20-10: veinte días de permanencia en la escuela y diez de estadía en sus hogares. Posibilita la convivencia de niños que viven cerca de la escuela y se trasladan diariamente con algunos que pernoctan en la misma. En general, se han constituido desde la normativa como "experiencia educativa".

El Ministerio de Educación de la Nación describe que "el desafío para los docentes es programar la enseñanza de modo de sostener la continuidad de los aprendizajes a través de actividades propuestas a los alumnos para los períodos de estadía" (MEN, 2012, p. 17).

Según las características del medio geográfico donde docentes y alumnos no puedan asistir regularmente a la escuela en el período del año que se establece como ciclo lectivo, cada jurisdicción provincial adopta medidas para garantizar la continuidad pedagógica como considerar "períodos especiales". Estas consisten en una suspensión más extensa que para el conjunto de escuelas durante el período invernal. 
La mayoría de los partidos del interior de la provincia de Buenos Aires cuentan con cientos de kilómetros de caminos de tierra. Durante el período invernal se pueden volver intransitables cuando las precipitaciones son continuas en el tiempo. Esto imposibilita que los docentes puedan llegar hasta los establecimientos educativos, restando días de clases a los alumnos que viven en el campo. En el último quinquenio hubo años donde esta reducción superó incluso el 20\% de los días hábiles.

\subsection{Estado del arte de la dinámica sociodemográfica y educativa en los espacios rurales en Maipú y la región.}

La evolución de las escuelas rurales y su dinámica en Maipú y en la región tiene algunos antecedentes de trabajos de investigación realizados fundamentalmente en la última década. Los temas estudiados fueron la evolución demográfica de los espacios rurales, las características del sistema educativo y el papel de las tecnologías de la comunicación en la vida escolar y cotidiana.

Annessi (2010) estudió la dinámica sociodemográfica de la población rural de Maipú desde mediados del siglo XIX hasta inicios del siglo XXI. Además, analizó la distribución espacial, la estructura demográfica por edad y sexo, las actividades del sistema agrario y la calidad de vida de la población rural. También Annessi (2016) hace un estudio pormenorizado del sistema educativo de la Región Educativa $\mathrm{N}^{0} 18^{1} \mathrm{con}$ especial énfasis en el caso de Maipú, donde analiza los establecimientos educativos y la matrícula de acuerdo con su localización geográfica (urbana o rural), y por tipo de gestión (pública o privada).

Más adelante, Bachex (2017) analiza la evolución de la matrícula en las escuelas rurales dispersas de los distritos de Maipú y General Guido (pertenecientes a la Región Educativa N¹8), para el período 2008-2017. Además, realiza un estudio pormenorizado de la localización de los alumnos en relación a la escuela a la que asisten para identificar medios de transporte más utilizados, distancia recorrida, como así también de los docentes. Con el uso de un Sistema de Información Geográfico se mide la efectividad de los servicios educativos en cuanto a distancia a la localización de los alumnos a través de cálculos de áreas de influencia con el uso de la herramienta de buffer.

Annessi, Bachex y Demirta (2018) profundizan los estudios anteriores al analizar la dinámica demográfica de la población rural y de manera simultánea la oferta educativa rural en dicho territorio. En esta investigación se estudia el constante proceso de despoblamiento rural dado durante las últimas cuatro décadas, y que de no ser por el sostenimiento e incluso creación de nuevas escuelas primarias, secundarias y jardines de infantes en este espacio, estaría prácticamente sin habitantes. También abordan algunas de las últimas medidas políticas que afectaron de manera decidida a los espacios rurales que, utilizando como argumento la "optimización de recursos", llevaron a que varios establecimientos educativos se cierren, sin pensar en el valor que generan dichas instituciones en el territorio.

Los usos, apropiaciones y prácticas de las telecomunicaciones y los medios que realizan las poblaciones rurales de los partidos de Maipú, General Guido y Ayacucho fueron analizados por Acosta (2019a). El autor estudia de qué manera los habitantes

1 Mapa de Regiones Educativas de la Provincia de Buenos Aires, Argentina: https://bit.ly/3gswLmL 
del espacio rural otorgan sentido a los servicios de telefonía, TV satelital e internet; y cómo los insertan en su vida cotidiana. El trabajo profundiza el papel jugado por las escuelas rurales, instituciones fundamentales que permiten la escolarización de los hijos de los empleados rurales. Las personas que viven en zonas rurales poseen distintos niveles de apropiación de las tecnologías de comunicación. Existen quienes utilizan el teléfono móvil para llevar las cuentas del establecimiento y otros que la llamada de voz es la acción más eficaz de comunicación con sus familiares.

En un trabajo de investigación realizado a pocas semanas de iniciada la cuarentena, Annessi y Varettoni (2020) indagaron a docentes de Ayacucho y Maipú acerca de las propuestas de enseñanza y medios de comunicación que han decidido emplear para atender a la continuidad pedagógica en la emergencia sanitaria y de qué manera tuvieron que realizar ajustes a las tareas que originalmente tenían planificadas para el aula, a la vez que predominan formas de interacción mediadas por recursos digitales. Algunas de las conclusiones obtenidas en este estudio dan cuenta de la complejidad y la diversidad de experiencias desarrolladas por los docentes.

\subsection{El ámbito de estudio: escuelas, educación y espacio rural de Maipú}

El partido de Maipú está localizado en el sudeste de la provincia de Buenos Aires, y se encuentra en la Región Educativa $N^{\circ} 18$, una de las 25 en que se divide esta provincia. Para 2010 contaba con 10.188 habitantes, con un 87,2\% que residía en Maipú, la ciudad cabecera del partido. El resto se distribuye en las localidades rurales de Las Armas y Santo Domingo, y dispersa por el espacio rural.

La localidad de Las Armas para el último censo contaba con 495 habitantes y Santo Domingo con 95. La población dispersa en el espacio rural era de 845 habitantes. En los tres casos hubo descensos para el último período intercensal 2001-2010, de $15,3 \%, 30,6 \%$ y $24,8 \%$, respectivamente.

En el período 2008-2020 la matrícula rural muestra una reducción como consecuencia de los procesos globales de despoblamiento. Se suman también a los cambios permanentes en los empleados asalariados o jornaleros rurales que presentan una alta movilidad laboral, cambiando de empleo con elevada frecuencia.

La mayoría de los alumnos/as se dirigen a la escuela en movilidad propia o son llevados por su familia en vehículo particular, incluso algunos se trasladan a caballo. En otros casos la maestra los lleva en su automóvil camino a la escuela. La distancia recorrida por un alumno rural de este partido es en promedio de siete kilómetros diarios.

Annessi, Bachex y Demirta (2018) describen que las distancias recorridas por los docentes se extienden desde los 10 a los 67 kilómetros. En promedio, un docente rural de Maipú recorre 43,7 kilómetros para llegar a su escuela. La mayor parte del trayecto se transita por caminos de tierra.

Maipú cuenta con un total de ocho escuelas primarias localizadas en el espacio rural. Seis de ellas se hallan en el espacio rural disperso, y sólo dos en localidades como Santo Domingo y Las Armas.

Estas dos últimas son las escuelas con mayor matrícula de alumnos, con 17 y 81 respectivamente. El resto tienen entre 3 y 9 alumnos. A continuación, se presentan las matrículas y secciones de cada establecimiento educativo. 
Tabla 1. Establecimientos y matrícula de escuelas primarias rurales, partido de Maipú.

\begin{tabular}{|c|c|c|}
\hline Establecimiento & Cantidad se secciones & $\begin{array}{l}\text { Matrícula Inicial } 2020 \text {, } \\
\text { al primer día de clases. }\end{array}$ \\
\hline$E P N^{\circ} 4$ & 1 & 6 \\
\hline$E P N^{\circ} 5$ & 1 & 9 \\
\hline EP N ${ }^{\circ} 7$ & 2 & 17 \\
\hline$E P N^{\circ} 9$ & 1 & 3 \\
\hline$E P N^{\circ} 13$ & 5 & 81 \\
\hline EP N ${ }^{\circ} 15$ & 1 & 5 \\
\hline$E P N^{\circ} 16$ & 1 & 5 \\
\hline $\mathrm{EP} \mathrm{N}^{\circ} 17$ & 1 & 5 \\
\hline
\end{tabular}

Fuente: Elaboración propia, con datos de la Jefatura de Inspección Distrital de Maipú.

Mapa 1. Localización de escuelas primarias rurales. Partido de Maipú

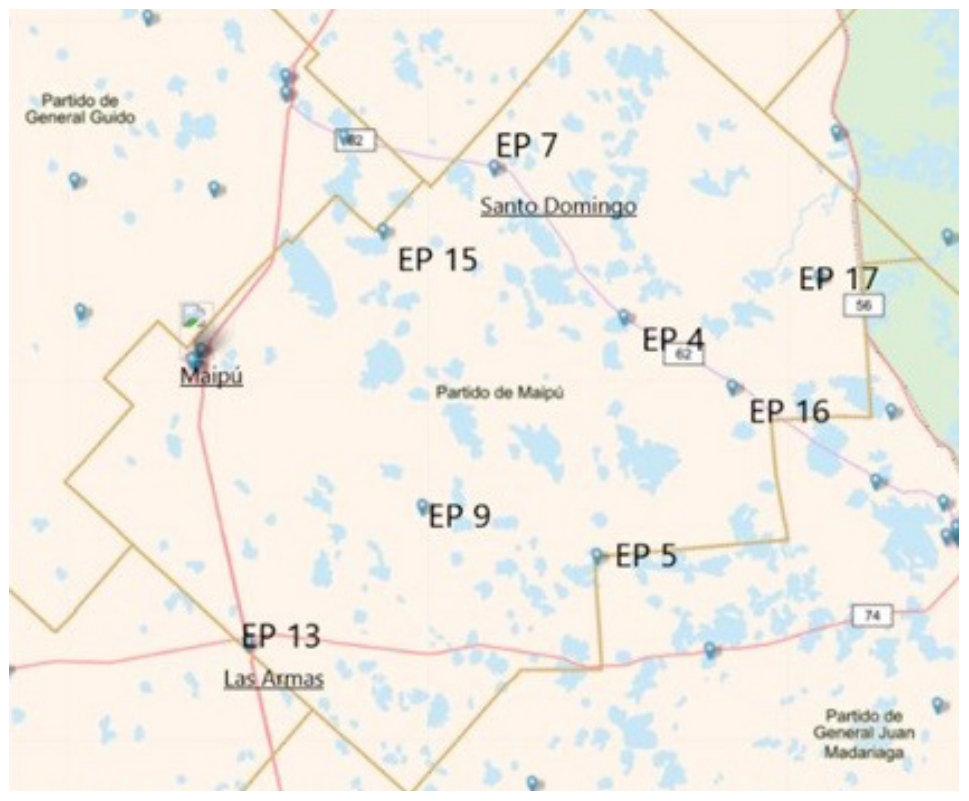

Fuente: Elaboración propia.

\subsection{Estrategias docentes en este escenario de pandemia.}

El decreto 297/20 estableció el ASPO debido a la pandemia de la COVID-19. Todas las instituciones de todos los niveles de la educación básica se cerraron el lunes 16 de marzo y no se sabe -al momento de producir este documento- hasta qué fecha permanecerá esta situación. 
De inmediato al anuncio del confinamiento obligatorio se configuró un sistema de enseñanza en emergencia en el que todos los actores (autoridades políticas y educativas, equipos de gestión y docentes) se vieron involucrados para desarrollar y hacer llegar las actividades y materiales a los hogares de los alumnos.

Ellos vieron profundamente transformada su vida cotidiana, tanto por el contexto inédito general como por el compromiso que debían asumir respecto a la colaboración y el seguimiento del trabajo escolar.

Frente a este escenario los docentes ejercieron una de las tareas centrales de la enseñanza: la planificación. En otras palabras, anticipar cómo se pretende desarrollar el trabajo con cada contenido en el aula constituye una instancia relevante de la práctica y se asume como un momento de producción intelectual.

Terigi (2012) advierte que esas decisiones se fundamentan, producen y reproducen en las prácticas atendiendo a un saber pedagógico por defecto, el cual es estructurado por características y condiciones que atraviesan la organización de la mayoría de las instituciones de la educación básica como la presencialidad, la simultaneidad, la clasificación por edades, la descontextualización, entre otros. A la vez menciona que ese saber circula en los espacios de formación y se reproduce en las prácticas llevando a que, en aquellos escenarios menos usuales (por ejemplo, escuelas con aulas plurigrado o con modalidad a distancia o semipresencial), los docentes se reconozcan menos capaces para desarrollar su tarea.

Sabemos que no todas las escuelas tienen esa organización por defecto, como es el caso de la mayoría de las escuelas primarias rurales. A su vez, año a año se generan planes educativos que se estructuran a partir de otras configuraciones (por ejemplo, la educación domiciliaria y hospitalaria, los programas de terminalidad del nivel primario o del secundario, entre otros).

Esto demanda desafíos profesionales para actuar en esos otros escenarios (planificar para alumnos que comparten una misma aula, pero pertenecen a grados distintos en un plurigrado, preparar materiales para alumnos que no están presentes en el aula por un largo período, etc.) que, sin duda, interpelan la formación -tanto inicial como continua- y quizás las decisiones se vayan construyendo y fundamentando a partir de lo que Terigi (2006; 2008) denomina "saber hacer" . Esto es desde sus propias decisiones, prácticas y experiencias en esas "otras" escuelas (ibídem).

La situación excepcional por la que se atraviesa un tanto se corresponde con estas últimas demandas y desafíos. Se suma la diferencia que se constituyó en una generalidad, más allá de la organización institucional, su ubicación y nivel educativo al que pertenece, y prácticamente configurada en dos o tres días, inmediatamente después que se comunicó la emergencia sanitaria.

Los docentes de manera acelerada tuvieron que repensar sus planificaciones y recursos para atender a estas exigencias. Se requirió preparar propuestas de enseñanza que puedan ser acercadas a los hogares involucrando la previsión y búsqueda de medios para atender a la enorme diversidad y variantes necesarias. 
La conectividad colabora mucho en la comunicación y acceso, pero también es un recurso que tiene un costo elevado y en algunas zonas, por más que se desee y se tengan los recursos para acceder, no existe por falta de infraestructura (como en muchas pequeñas localidades o establecimientos rurales) (Annessi y Varettoni, 2020, p. 61).

Un indicador central para la elaboración del "plan de continuidad pedagógica" estuvo puesto en el grado de comunicación que han logrado establecer en años anteriores con los hogares.

Estas reflexiones ponen en evidencia que la responsabilidad que debieron asumir los docentes va más allá de cómo conectarse con los hogares. El mayor desafío es de qué manera reconstruir los sentimientos e interacciones que se producen en el seno de la escuela, a la vez que las decisiones pedagógicas requieren contemplar otras dimensiones que las que se ponen en juego en la cotidianeidad del aula.

El plantel docente de las escuelas rurales, a diferencia de las urbanas, conocen con mayor precisión los tiempos de los alumnos, de las familias y de las actividades. El docente rural conoce la idiosincrasia, la hace propia y planifica en consecuencia, y debe desempeñar simultáneamente muchas funciones. Sabe que todo momento es un espacio de enseñanza, que cada uno es importante para el niño, y que es mejor aprovecharlo porque se podrá ver interrumpida momentáneamente por la próxima lluvia o definitivamente por la pérdida del trabajo del jefe del hogar y su traslado hacia otro sitio, con la consecuente búsqueda de una nueva escuela: "Es así que el docente rural quizás enriquece su perspectiva al considerar el tiempo, su tiempo y el del niño, como único y determinante" (DGCyE, 2007, p. 9).

El recorrido en este tipo de establecimientos educativos les permitió adquirir a través de la experiencia unas capacidades específicas para desplegar estrategias donde su presencia física no sea posible. Esto les permitió enfrentar mejor esta situación excepcional.

Una de las estrategias aplicadas se aprecia desde el campo de la comunicación, entendida como el lugar de producción de significados y disputas de sentido. Según el educador Paulo Freire, la comunicación implica dialogicidad y no hay sujetos pasivos en este proceso (Freire, 1973). En un escenario donde el acceso a internet móvil y fijo es un bien escaso, los docentes se adecuaron para mantener el contacto con sus alumnos y sus familias. El uso del teléfono móvil cumple un rol clave en esta tarea.

Los docentes desarrollaron un proceso particular de apropiación y/o uso, entendiendo a estos como "un proceso tanto material como simbólico en el que los individuos toman el contenido significativo de un artefacto y lo hacen propio, confiriéndole sentido e incorporándolo en las prácticas y relaciones cotidianas" (Acosta, 2019b, p. 4).

Thompson (1998) define a la apropiación como una rutina donde "los individuos se implican y trabajan con los materiales simbólicos que reciben" (p. 62). El uso de las tecnologías es un proceso cargado de conocimientos -saber hacer y poder hacer-, de hábitos y reglas culturales, de un "aire simbólico" (Boczkowski y Mitchelstein, 2017) de significaciones y sentidos en torno a nuestra vida cotidiana. 
Ruelas (2014) retoma los dichos de Rheingold (2002) al afirmar que el "real impacto de las comunicaciones móviles vendrá no de la tecnología misma, sino de cómo la gente la utiliza" (p. 107). Rheingold las denomina smart mobs, es decir, dispositivos que son inteligentes a medida que las personas las adaptan a sus propias necesidades, para sus redes sociales y la acción colectiva. Para Ruelas, el teléfono móvil se transforma en un "aparato vestible, ubicuo, multifuncional, para comprar y vender, entretenerse, y también para conflictuarse” (Ruelas, 2014, p. 107).

La educación es un proceso de formación de sujetos, subjetividades e identidades sociales y la comunicación un proceso de producción social de sentidos y significados. Hacer docencia es también saber comunicar. Los docentes combinaron dos tipos de conocimiento en este período: el saber hacer de su experiencia en la educación en espacios rurales y el saber usar proveniente de las capacidades y limitaciones que los dispositivos tecnológicos permiten.

\section{Preguntas de investigación, métodos y materiales}

El trabajo se basó en una investigación cualitativa atenta a las dinámicas y procesos que están presentes en el objeto de estudio. Denzin y Lincoln (1994, p. 2) define a la investigación cualitativa como aquella que "incluye la recolección y el uso estudiado de una variedad de materiales empíricos -estudios de caso, experiencia personal (...) entrevistas (...)- que describen la rutina, los momentos problemáticos y los significados en la vida de los individuos".

Para ahondar en las prácticas y reflexiones de los docentes se realizaron entrevistas a seis docentes de las ocho escuelas rurales. Ellos cumplen simultáneamente con la función de directoras. Debido al ASPO, las entrevistas se realizaron a través de llamadas de voz y con acuerdo previo mediante WhatsApp. En aquellos casos donde se necesitaba profundizar alguna respuesta, se logró a través del mismo instrumento.

Yuni y Claudio Urbano definen a la entrevista como una herramienta que le "permite al investigador acceder a hechos, descripciones de situaciones o interpretaciones de sucesos o fenómenos (internos o externos) a los que no se podría acceder de otro modo" (Yuni y Urbano, 2014, p. 82).

Los ejes de indagación apuntaron en dos direcciones complementarias. El primero sobre lo que estaba sucediendo en la primera etapa del ASPO, con foco en las características de los módulos de continuidad pedagógica para los alumnos. El segundo abordó sobre qué canales de comunicación utilizaron los equipos de supervisión (inspectores), los alumnos y sus familias.

Las preguntas que guiaron esta investigación abordaron las particularidades de los módulos propuestos para la enseñanza de los alumnos, los cambios o continuidades en las características de las actividades en relación a años anteriores, los objetivos propuestos habitualmente durante el año, el tipo de estrategias de los recursos empleados, la función asignada a las tecnologías educativas, la retroalimentación con los alumnos, los medios de comunicarse con ellos, sus familias y los equipos de supervisión.

Los docentes/directivos entrevistados pertenecen a los siguientes establecimientos educativos: 
- María José, docente y directora de la EP N4 (Segurola).

- Virginia, docente y directora de la EP N5 (San Simón).

- Lorena, docente y directora de la EP N7 (localidad de Santo Domingo).

- Juliana, docente y directora de la EP N9 (Santa Isabel).

- Lorena, docente y directora de la EP N¹5 (Yamahuida).

- Luciana, docente y directora de la EP N¹6 (Monsalvo).

El cuestionario constó de las siguientes preguntas:

- ¿Cómo se adaptaron a esta situación de ASPO?

- ¿Cómo planificaron la entrega de los módulos de continuidad pedagógica?

- ¿Qué materiales recibieron por parte de las autoridades de educación municipales, provinciales y nacionales?

- ¿Qué ocurrió con el servicio de comedor?

- ¿Cómo se comunican con los alumnos?

- ¿En qué horarios y días se comunican?

- ¿Los contenidos son los mismos a los planificados antes del inicio de la cuarentena?

- ¿Son temas nuevos?

- ¿Cómo abordaron el tema de la COVID-19?

- ¿Cómo recibieron estos cambios los alumnos?

- ¿Cómo la recibieron los padres?

Una vez desgrabada y ordenada la información correspondiente se procedió a la descripción y análisis. Los datos fueron organizados en siete categorías distinguidas con subtítulos. Los resultados se desarrollan a través de la citación y argumentación, técnica que Gomes (2003) considera útil para agrupar elementos, ideas o expresiones y puede servir para cualquier tipo de análisis en investigación cualitativa.

\section{Resultados}

Se presentan los resultados de las entrevistas realizadas a los docentes a dos meses de haberse iniciado el período de ASPO.

\subsection{Estrategias durante el ASPO}

Las escuelas rurales suspendieron las clases presenciales en la tercera semana de marzo. A partir de ese momento los docentes entregan módulos de continuidad pedagógica a sus alumnos cada quince días. Esta serie de módulos están impresos en papel y contienen todas las áreas, Matemática, Prácticas del Lenguaje, Ciencias Naturales y Ciencias Sociales. Los profesores de asignaturas específicas como Música, Artística o Educación Física también envían sus tareas.

La acción de los docentes rurales puede distinguirse en dos fases. La primera ocurrió durante la primera semana luego de dictada la suspensión de las clases presenciales. Los docentes entregaron contenidos de emergencia para no perder el proceso educativo, con la idea de volver en un tiempo breve. 
La segunda etapa se desenvuelve con la extensión del ASPO. La educación se desarrolla de manera planificada y los módulos de continuidad pedagógica forman parte de la nueva normalidad. Los módulos elaborados por los docentes, pero también aquellos elaborados por los ministerios de educación provincial y nacional, son llevados por los docentes a las estancias de sus alumnos, o bien son los padres quienes se dirigen a la escuela para buscarlos. Esto depende de cada institución y de la localización del alumno.

El proceso se realiza cada quince días y es un intercambio. Los docentes entregan los contenidos nuevos y retiran los realizados para su corrección. Asimismo, proporcionan a los alumnos bolsones de alimentos como reemplazo del servicio de desayuno, que se brinda habitualmente en la escuela.

La entrega de módulos de continuidad pedagógica es una práctica arraigada. Todos los años los alumnos reciben distintos módulos para aquellos días de lluvia y que imposibilita asistir a la escuela. No obstante, la pandemia modificó en cierto grado los planes pedagógicos, pasando a clases asincrónicas en tiempo y espacio. Los alumnos completan las actividades en sus casas hasta donde pueden, y si se encuentran con alguna consigna que no entendieron, la explicación de la maestra llega a través de WhatsApp o llamada de voz.

\subsection{Las características de los contenidos}

En un primer momento los contenidos dados no son nuevos, sino que son actividades de repaso, pero con un grado mayor de dificultad. "Si en primer grado aprendieron sobre el desplazamiento de los animales, en segundo están viendo los tipos de pelaje. Es un trabajo de extensión y ampliación de conocimientos", explica Natalia, docente de la EP N¹5 de Yamahuida.

El objetivo es desarrollar la capacidad exploratoria de los alumnos. En el mismo sentido, los alumnos aprenden la herramienta de creación de cuadros sinópticos o la de observación. La premisa es que, ante la ausencia de una persona con una preparación pedagógica y calificada para enseñar, la tarea no puede recaer sobre los padres.

No son temas nuevos porque yo no puedo ponerles la responsabilidad a las familias de que enseñen. Las familias no tienen la obligación de enseñar. Sí de acompañar y de compartir las prácticas pedagógicas como lo haríamos si fuéramos a la escuela (Natalia, docente de la EP N¹5).

María José, de la EP $N^{\circ} 4$, detalla que la mayor dificultad de aprendizaje se encuentra en matemáticas. Es una asignatura que depende mucho de la bajada a tierra con el pizarrón, los ejemplos y la conversación junto al grupo. Los audios o explicaciones a través de las redes sociales a veces no alcanzan.

Los docentes debieron en algún momento modificar sus estrategias e incorporar juegos didácticos por tener escasas respuestas por parte de los alumnos. Además, entregaron libros que forman parte de las bibliotecas escolares y elementos para pintar, colorear y dibujar. 


\subsection{La COVID-19 como tema de relieve}

La temática de la COVID-19 fue abordada a través de distintos módulos con información para que pudiesen comprender qué ocurre en el país con la pandemia, los orígenes de esta nueva enfermedad, la forma de evitar el contagio y el porqué del ASPO.

También se apela a la creatividad, como en la escuela $N^{\circ} 7$ que realizaron un video destacando los cuidados de higiene y limpieza para contrarrestar al virus, que luego socializaron con sus familias. Virginia, docente de la escuela $\mathrm{N}^{\circ} 5$, relata que en su institución "el equipo de psicología brindó una charla con respecto al Coronavirus".

Luciana, de la EP $N^{\circ} 16$, optó por mostrarle distintas fotografías de la ciudad cabecera de Maipú, acerca de cómo las personas utilizaban tapabocas, formaban fila afuera con un metro de distancia para entrar a un comercio, o cómo las calles con acceso a la carretera estaban cerradas con montículos de tierra.

En la segunda recorrida fuimos con los voluntarios de la COVID, que son chicos que trabajan en el hospital municipal. Ellos contaron la diferencia entre urgencia y emergencia, quién puede ir a la salita y quién no, porque se había desdibujado esa idea errada de que nadie podía ir al hospital. Había situaciones en las que había un dolor fuerte y no habían ido al hospital por miedo a contagiarse (Lorena, docente de la EP $\mathrm{N}^{\circ} 7$ ).

\subsection{Alumnos y padres: la relación con los docentes}

Los docentes reconocen el compromiso demostrado por los padres en este contexto, desde ayudar a sus hijos con las actividades, hasta limpiar y ventilar la escuela. Es por ello que dentro de los módulos hay actividades con consignas "para conversar en familia", "para que me ayuden en casa" o "para jugar todos juntos".

"Muchas mamás se sientan con ellos a realizar las tareas", dice Virginia. A veces los hermanos mayores son quienes ayudan a los más pequeños: esta es una característica de la educación rural con o sin pandemia. Los padres colaboran con los docentes porque la escuela es la institución más importante de toda comunidad rural. Se produce allí una educación a nivel horizontal entre docente-padre-alumno.

La entrega de módulos en la escuela o en los hogares es un espacio de encuentro entre los docentes y los padres. Allí se despejan las dudas que fueron surgiendo y las consultas de los nuevos contenidos. Este contacto funciona también para socializar las novedades de la ciudad y la región.

\subsection{El celular como dispositivo de encuentro y consultas}

Posteriormente a la entrega de los módulos, la comunicación continua a través de WhatsApp. Cada docente establece con sus alumnos los términos para mantenerse comunicados y cómo será la revisión de los contenidos. Cada maestra se organiza de acuerdo con las posibilidades y obstáculos de cada alumno.

En la mayoría de los casos los teléfonos son propiedad de los padres y los docentes les envían videos cortos como complemento de los módulos. Mientras hay alumnos que en sus casas cuentan con conectividad, otros en cambio no. 
En el primero de los casos la dinámica de comunicación es diaria y fluida a través de los grupos de WhatsApp. Lorena detalla el modus operandi de la siguiente manera:

Diariamente vamos corrigiendo algunas actividades, vamos subiendo cosas. Por ejemplo, en Sociales estábamos trabajando unas actividades de Provincia y les voy subiendo algún video cortito, que sea uno por día y que puedan descargar para no consumirle los datos. Que sea entretenido y fácil para que puedan resolverlo (Lorena, docente de la EP $N^{0} 7$ ).

La respuesta de los padres son mensajes con textos, fotos y videos de sus hijos realizando los deberes, o audios con preguntas sobre cómo se resuelve tal actividad.

Este uso específico del WhatsApp habilita pensar a las tecnologías como un territorio donde los usos esperables pueden modificarse o no ocurrir en un contexto particular. Por el contrario, son condición de posibilidad de nuevas prácticas y luchas por el sentido.

Williams (1997) establece que existen tensiones en el uso de una tecnología, entre lo que él denomina usos dominantes, residuales y emergentes. Lo dominante es lo que se encuentra legitimado; lo residual es aquello que pertenece al pasado, pero opera en el presente; y lo emergente refiere a los nuevos usos, prácticas y apropiaciones que es alternativa u oposición de lo dominante.

Los tiempos para hacer consultas e intercambios entre alumnos-padres-docentes en la mayoría de los casos es durante todo el día. Los docentes están atentas al celular, conscientes de esta situación:

Yo estoy siempre disponible. A veces son las ocho de la mañana, porque la vida en el campo empieza muy temprano, y por ahí me escriben 'Seño, ¿estás?', y yo les respondo 'Sí, estoy'. He vivido en el campo y sé lo que es esa realidad. Con la otra familia, recién a las ocho llega el papá que tiene el teléfono, que es el que ha andado en la estancia. Entonces por ahí ha podido conectarse y baja los mensajitos o trata de ver algún video para después contarlo. Si tienen dudas, recién a las ocho de la noche, se comunican. Aunque tratan de no hacerlo en lo posible (Natalia, docente de la EP $\mathrm{N}^{\circ} 15$ ).

En el caso de Juliana, de la EP $N^{\circ} 9$ de Santa Isabel, el horario estipulado para comunicarse es a la mañana de 8:30 a 12:30, como en un día de clase: "Lo mismo ocurre con las áreas especiales. Se respetan días y horarios. Si surge alguna consulta fuera del mismo, obviamente que también se atiende en otros horarios".

Los padres, propietarios de los teléfonos, no cesaron sus compromisos laborales. Ala poca señal para comunicarse, se le suma su disponibilidad horaria. Generalmente utilizan la mañana y la noche para descargar los contenidos multimedia.

(Los padres) me escriben a la nochecita, yo veo el mensaje y al otro día les contesto, porque hay un solo celular en la casa que tiene datos y WhatsApp, que es del padre. Ellos a la mañana lo ven y lo resuelven. $Y$ si tienen alguna duda, a la noche me vuelven a escribir. O me escriben a la tarde y se les manda el mensaje cuando enganchan señal en algún momento (María José, docente de la EP N4). 


\subsection{Casos particulares}

Hay situaciones favorecedoras del proceso de enseñanza, como es la situación de los alumnos de la escuela de Monsalvo, donde cinco de los seis alumnos cuentan con internet, computadora (cedida por la docente para este contexto) e impresora en el chalet de la estancia. Allí se comunican más asiduamente por redes sociales y es posible utilizar la plataforma de videollamadas Zoom.

La escuela cuenta con un Aula Digital Móvil entregada hace 5 años por el gobierno nacional y/o provincial con cinco netbooks, pero ninguna funciona, situación recurrente y habitual, que en caso contrario podría haber resuelto esta situación con los alumnos. La impresora forma parte de las Aulas Digitales Móviles, plan de infraestructura digital encabezado por el Ministerio de Educación de la Nación en el período 2015-2019.

Una situación parecida sucede en la escuela de Segurola, donde cada alumno posee una netbook en su casa, y de esta manera facilita el trabajo del docente porque en un pendrive les deja copiados los módulos, videos, audiolibros y libros digitales.

\subsection{La escuela presencial como espacio de sociabilidad}

La escuela es algo fundamental en sus vidas, es el eje de la vida social y comunitaria, y que no esté, como que rompe un poco con los modos de comunicación y sus rutinas. Es a través de las escuelas que ellos se enteran de las cosas. En la anterior recorrida llevamos todas las semillas de Pro Huerta ${ }^{2}$. No habían sembrado porque nosotras no habíamos llegado. Ese rol que cumple la institución en la sociedad es muy fuerte. Están esperándonos para encontrar respuestas que muchas veces no tenemos (Lorena, docente de la EP N07).

Los chicos desean poder reencontrarse con sus compañeros y sus docentes. Ellos no pueden ir al pueblo ni estar con sus amigos que están a varios kilómetros de distancia. La escuela es el punto de encuentro con sus compañeros. A los padres les ocurre lo mismo. El ingreso y egreso a la escuela es un espacio de sociabilidad.

Fernández y Magnani (2020) enuncian que la escuela sufrió un "cimbronazo drástico" durante la pandemia. La escuela, prosiguen, vio en jaque "el encuadre que la caracteriza desde hace siglos: un tiempo y espacio de encuentro de cuerpos presentes. El mundo digital ofreció alternativas vitales para salir a flote durante la cuarentena y mantener la relación con el mundo educativo" (Fernández y Magnani, 2000, párr. 5).

\section{Conclusiones}

El presente trabajo describe y analiza los resultados de entrevistas realizadas a docentes de escuelas primarias de las zonas rurales de Maipú, provincia de Buenos Aires. El interés estuvo en conocer qué propuestas de enseñanza y estrategias se pusieron de relieve para sostener la continuidad pedagógica durante el ASPO iniciado en marzo de 2020.

\footnotetext{
${ }^{2}$ Para ampliar información sobre el Programa Pro Huerta: https://inta.gob.ar/documentos/prohuerta.
} 
Las escuelas rurales interpelan a las urbanas porque han desarrollado con el tiempo una dinámica que les ha permitido hacer frente a este escenario con mayor rapidez. Los docentes de las escuelas estaban acostumbrados a trabajar con las familias, donde ésta ya forma parte de la escuela, con tiempos prolongados de no presencialidad, situación inédita en cambio para todas las escuelas urbanas.

La estrategia de entrega de módulos cada quince días empleada durante el ASPO es una práctica que está articulada y entrenada debido a los frecuentes períodos de días de lluvia y la imposibilidad de acceso a los establecimientos educativos por el estado de los caminos de tierra. Por lo tanto, las medidas adoptadas desde el inicio de esta pandemia de continuidad pedagógica no resultaron ajenas para docentes, alumnos y padres.

El celular como dispositivo tecnológico funcionó como un espacio de comunicación para este trinomio en aquellos casos que lo permitía. El uso del celular entre los docentes-alumnos-padres habilita un lugar de conversaciones para realizar consultas sobre las tareas y ponerse al día con respecto a las cotidianeidades de cada uno.

No obstante, esto no quiere decir que el proceso enseñanza-aprendizaje se desarrolle con normalidad. Nunca antes había sido tan prolongado. Nunca antes se había extendido por casi un ciclo lectivo completo. No hay modo de reemplazar las clases presenciales. La explicación junto al pizarrón, la ejemplificación y la interacción entre la docente y los alumnos. En este punto, los módulos de continuidad pedagógica entregados quincenalmente solo permiten transitar este período de aislamiento garantizando que los alumnos sigan aprendiendo aquellos contenidos prioritarios.

En los espacios rurales hay situaciones muy heterogéneas porque no todas las familias cuentan con el tiempo ni las competencias necesarias para poder acompañar la continuidad pedagógica. En estas situaciones se vuelve difícil sostener la educación a distancia, debido a la escasa o nula señal de Internet.

La nueva normalidad que se nos presenta implica poner en cuestión discursos y conceptos previos: la relación padres-docentes, la escuela como institución, la presencia de la tecnología como solución mágica. Este nuevo escenario nos lleva a caminar a tientas, con avances y retrocesos, y nos desafía a no paralizarnos, a afrontar esta situación con las mejores herramientas posibles: es un desafío que los docentes de escuelas rurales han aceptado y lo están logrando. Esto es posible porque el acuerdo tripartito entre docentes, alumnos y padres está muy consolidado en términos de compromiso, responsabilidad y cooperación.

En ese sentido el presente estudio conjuga la descripción y el análisis de la situación de las escuelas rurales desde una perspectiva integradora: el saber hacer de los docentes desde una perspectiva educativa y el saber usar proveniente de los estudios de comunicación ligados a la apropiación social de los dispositivos tecnológicos.

La continuidad pedagógica de las escuelas rurales se desarrolló con prácticas planificadas y aplicadas con anterioridad. Las restricciones como consecuencia de la pandemia hicieron que estas acciones se volviesen a implementar, no sin antes adecuarlas al nuevo escenario. 


\section{Referencias}

Acosta, J. (2019a). Usos de las Telecomunicaciones y Medios en poblaciones rurales: descripción y análisis de las apropiaciones culturales en los partidos de Maipú, General Guido y Ayacucho. Trabajo Integrador Final. Tesis de Licenciatura en Comunicación Social. Facultad de Periodismo y Comunicación Social. Universidad Nacional de La Plata. Buenos Aires, Argentina. Recuperado de: https://bit.ly/3xdKmoB.

Acosta, J. (2019b). Usos y apropiaciones de la telefonía móvil. El caso de las escuelas rurales del partido de Maipú. Actas de Periodismo y Comunicación, 5(2). Facultad de Periodismo y Comunicación Social. Universidad Nacional de La Plata. Buenos Aires, Argentina. Recuperado de https://bit.ly/3vk03cC.

Annessi, G. (2010). Atlas social, demográfico y territorial del Maipú. Un aporte geográfico para su estudio. Escuela Normal Superior Normal EVA, Maipú.

Annessi, G. (2016). El sistema educativo de la Región Educativa N 18. En: Annessi, G. (Coord.). Voces de docentes. Maipú en el marco del Bicentenario, 49-72. Centro de Capacitación, Información e Investigación Educativa, Maipú, Argentina.

Annessi, G., Bachex, V. y Demirta, P. (2018). La oferta educativa en los espacios rurales como factor de retención demográfica. El caso de la región del este de la provincia de Buenos Aires. Maipú y General Guido 2008-2017. I Congreso Iberoamericano de Docentes. Modalidad Virtual, 26/11 al 08/12 de 2018, Algeciras, Cádiz. Recuperado de https://bit.ly/3vgcBld

Annessi, G. y Varettoni, M. (2020). Cuando el hogar y la escuela pasan a ser el mismo lugar. La tarea docente en tiempos de cuarentena. En: Novedades Educativas $N^{0} 355$, julio, 60-64.

Bachex, V. (2017). La oferta de la educación en los espacios rurales como factor de retención demográfica. El caso de la región del este de la provincia de Buenos Aires. ISFD № 170. En: $2^{\circ}$ jornada de Geografía de ISFD de la Región Nº 18.

Boczkowski, P. y Mitchelstein, E. (2017). Smartphone, el aire que respiro. Revista Anfibia. Recuperado de https://bit.ly/3iNNOOf.

Bortz, J.E. (2017). 1918: la grippe en Buenos Aires. La sociedad porteña en crisis. Americanía. Revista de Estudios Latinoamericanos, 6, 230-261. Recuperado de https://bit.ly/3gu4sEr

Denzin, N. y Lincoln Y. (1994). Introducción: ingresando al campo de la investigación cualitativa. Manual de Investigación Cualitativa. Cap. 1. pp. 1-28. Traducción de Mario Perrone. Recuperado de https://bit.ly/3wfLV5D.

DGCyE (2007). La tarea de Enseñar en el Ámbito Rural. Dirección Provincial de Educación Inicial. Dirección General de Cultura y Educación. Dirección de Espacios y Contextos de Desarrollo, La Plata .

DGCyE (2009). Programa de Educación para el Desarrollo Rural e Islas. Documento No 1. La Plata: Dirección General de Cultura y Educación.

Fernández, E. y Magnani, S. (2020). El único cable hacia el mundo. Revista Anfibia. Recuperado de: https://bit.ly/3znchnW.

Freire, P. (1973). ¿Extensión o comunicación? La concientización en el medio rural. Buenos Aires: Siglo XXI.

Gomes, R. (2003). El análisis de datos en la investigación cualitativa En M.C. de Souza (Org.) Investigación Social Teoría, método y creatividad (pp. 53-63). Buenos Aires: Impresiones Sud-América S.A. Recuperado de https://bit.ly/3cCtQqF

MEN (2012). Módulo La Educación en contextos rurales. Especialización Superior en Educación Rural. Ministerio de Educación de la Nación. Buenos Aires: DGCyE.

Rheingold, H. (2002). Smart mobs. The next social revolution. Cambridge, Inglaterra: Basic Book

Ruelas, A. M. (2014). El teléfono celular y los jóvenes sinaloenses. Adopción, usos y adaptaciones. Revista Comunicación y Sociedad, 101-131. Guadalajara, México: Universidad de Guadalajara. Recuperado de https://bit.ly/3wgfMup 
Terigi, F. (2012). La enseñanza como problema en la formación en el ejercicio profesional. En Birgin, A. Más allá de la capacitación. Debates acerca de la formación de los docentes en ejercicio. Buenos Aires, Argentina: Paidós.

Terigi, F. (2008). Organización de la enseñanza en los plurigrados de las escuelas rurales. Tesis de Maestría. Buenos Aires, Argentina: Facultad Latinoamericana de Ciencias Sociales. Recuperado de https://bit.ly/35cHbSk.

Terigi, F. (2006). Las "otras" primarias y el problema de la enseñanza. En Terigi, F. (comp.) Diez miradas sobre la escuela primaria, Buenos Aires: Siglo XXI editores.

Thompson, J. (1998). Los media y la modernidad: una teoría de los medios de comunicación. Buenos Aires, Argentina: Paidós.

Williams, R. (1980). Marxismo y Literatura. Barcelona: Ediciones Península.

Yuni, J. y Urbano, C. (2014). Técnicas para investigar y formular proyectos de investigación, Vol. 2. Córdoba, Argentina: Brujas. 